\title{
Papel de la hipertensión arterial en la comorbilidad de la cefalea crónica
}

\author{
H.P. Grebe, J. Nunes da Silva, L.M. Diogo-Sousa
}

\section{RESISTANCE TO PHARMACOLOGICAL PROPHYLAXIS IN HYPERTENSIVE HEADACHE PATIENTS}

\begin{abstract}
Summary. Introduction. The literature concerning the association between headache and arterial hypertension is very scarce, mostly prior to the actual IHS classification and based on very diverse methods, being therefore difficult to interpret. Objectives. To analyse the association between arterial hypertension and two types of chronic headache and to determine, if and how arterial hypertension may affect the outcome of prophylactic therapy for chronic headaches. Patients and methods. Sixty-four files of our headache outpatient clinic, chosen randomly among patients suffering from migraine or tensional type headache (TTH), were analysed retrospectively. Patients were considered hypertensive, if blood pressure was higher than normal several times without an acute crisis present. Resistance to treatment was defined as lack of benefit concerning number and/or intensity of crisis. Results. Age varied between 18 and 80 years, the mean age being 42,9(SD 13,86) years. Eighty-six percent were females. Twenty-nine patients suffered from TTH and 35 from migraine. Prevalence of hypertension was 35,9\% among all patients, 28,5\% among migraine patients, 44,8\% among patients with TTH. The prevalence of resistance to treatment was 39,8\%, 34,3\% and 41,3\%, respectively. Of the patients resistant to treatment $60 \%$ were hypertensive and $62,5 \%$ of the hypertensive patients showed resistance to therapy. Conclusions. Arterial hypertension was more prevalent in the study sample than would be expected in an equivalent sample of the general population. Hypertension was significantly more prevalent among patients resistant to therapy, as was resistance to treatment among hypertensive patients. We therefore conclude that there is a relevant comorbidity between chronic headache and hypertension and that arterial hypertension complicates the control of chronic headaches. [REV NEUROL 2001; 33: 119-22]
\end{abstract}

Key words. Arterial hypertension. Chronic headache. Comorbidity. Therapy resistance.

\section{INTRODUCCIÓN}

El concepto de comorbilidad es definido comola asociación, mayor de la que cabría esperar por coincidencia, de dos entidades nosológicas [1]. La comorbilidad tiene importancia en el diagnóstico, en la medida en que su presencia en una de las dos patologías asociadas hace aumentar la sospecha hacia la aparición de la otra. También tiene implicaciones terapéuticas, dado que puede ofrecer oportunidades terapéuticas, como la comorbilidad de la cefalea y la epilepsia, pero puede igualmente condicionar problemas terapéuticos.

En relación con la migraña, existe comorbilidad bien documentada con varias entidades patológicas. El riesgo de accidentes cerebrovasculares aumenta de dos a cuatro veces entre las personas con migraña. La prevalencia de la epilepsia en pacientes con migraña es diez veces mayor que en la población general y el riesgo de padecer migraña aumenta en 2,4 veces en los epilépticos. La migraña está relacionada con la depresión, aumentando el riesgo de esta última en 4,1 veces en la migraña y constituyendo la depresión, a su vez, un factor de riesgo para la migraña [1].

La literatura existente sobre la comorbilidad de la cefalea de tensión (CTT) es escasa. La hipertensión arterial (HTA) como comorbilidad de las cefaleas, ya sea de la migraña, ya sea de la CTT, está poco estudiada y presente en la literatura.

El objetivo de este trabajo es analizar la asociación entre la HTA y la cefalea crónica en pacientes de una consulta de cefaleas y la influencia de la HTA en la respuesta de la cefalea a la farmacoterapia.

Recibido: 23.03.01. Aceptadotras revisión externa sinmodificaciones: 05.05.01.

Consulta de Cefaleas. Servicio de Neurología. Hospital de la Universidad de Coimbra. Coimbra, Portugal.

Correspondencia: Dra. Lívia Maria Diogo Sousa. Serviço Neurologia I. Hospitais da Universidade de Coimbra.P-3049 Coimbra.E-mail: livia@clix.pt Ó 2001, REVISTA DE NEUROLOGÍA

\section{PACIENTES Y MÉTODOS}

Se llevó a cabo un estudio retrospectivo, analizando 64 historias clínicas de la consulta de cefaleas. La elección fue aleatoria hasta obtener por lo menos 30 historias de pacientes con migraña (M) y otros tanto con CTT. Los criterios de inclusión para el análisis se muestran en la tabla I.

Un paciente fue considerado hipertenso si aparecían varios registros de HTA independientes de las crisis de cefaleas. Para la clasificación se utilizó la escala JNC-V1 (Tabla II). En los casos en que hubo dudas, clasificamos al paciente en función de su valor sistólico. Este procedimiento nos pareció el más lógico, dado que varios estudios recientes apuntan hacia una mayor importancia de este valor en comparación con el valor diastólico.

Para evaluar la eventual influencia de la HTA en la respuesta terapéutica definimos la farmacorresistencia como la falta de alteración o aumento de la frecuencia o intensidad de las crisis bajo el tratamiento profiláctico con fármacos aprobados para el mismo.

Dado el componente de distensión vascular en la patogenia de la cefalea, calculamos la presión de pulso $(\mathrm{PP})$ de los pacientes, siendo $\mathrm{PP}=\mathrm{TA}$ sistólica - TA diastólica.

Se registró la existencia de otras enfermedades, siempre y cuando su diagnóstico estuviera documentado explícitamente en el historial clínico.

\section{RESULTADOS}

Entre las 64 historias analizadas, 29 presentaban CTT y 35 migraña (17 con aura y 18 sin aura). La distribución porcentual se muestra en la figura 1.

La edad de los pacientes varió entre 18 y 80 años, siendo la media de edad de $42,86( \pm 13,86)$ años y la mediana de 44 años. Tras establecer varios grupos según los diferentes tipos de cefalea, el patrón de edad fue el siguiente: CTT: edad media de 49,04 ( $(14,57)$ años, mediana de 50,5 años; M con aura (MA): edad media de 34,6 $( \pm 9,32)$ años, mediana de 36 años; M sin aura (MWA): edad media de 39,83 $( \pm 12,52)$ años, mediana de 44,5 años.

Las pacientes de sexo femenino constituían un $86 \%$ del total, con mayor predominio en la CTT $(89,3 \%)$ que en la M $(82,9 \%)$.

La prevalencia de la HTA en la muestra estudiada se ilustra en la figura 2. Presentaban HTA 23 pacientes, que correspondían a 10 de los 35 pacientes con M (3 MA y 7 MWA) y 13 de los 29 pacientes con CTT. Entre los pacientes farmacorresistentes $(n=25), 15$ eran hipertensos. La diferencia entre los pacientes farmacorresistentes y los pacientes que respondieron al tratamiento fue estadísticamente significativa $(\mathrm{p}=0,003)$, mientras que la diferencia de la prevalencia entre los dos tipos de cefalea no lo fue $(\mathrm{p}=0,277)$. 


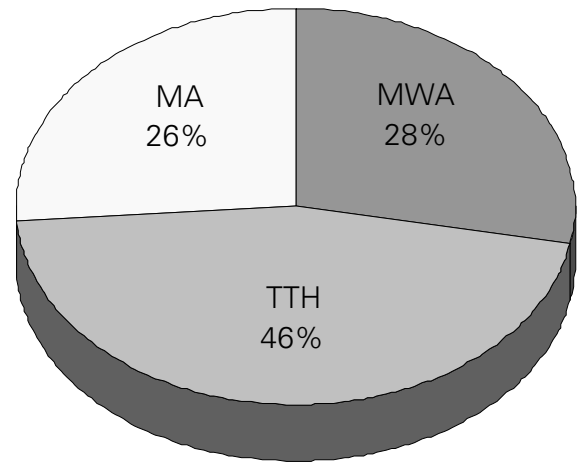

Figura 1. Distribución de los tipos de cefalea en la población estudiada. MWA: migraña sin aurea; MA: migraña con aurea; CTT: cefalea de tipo tensional.

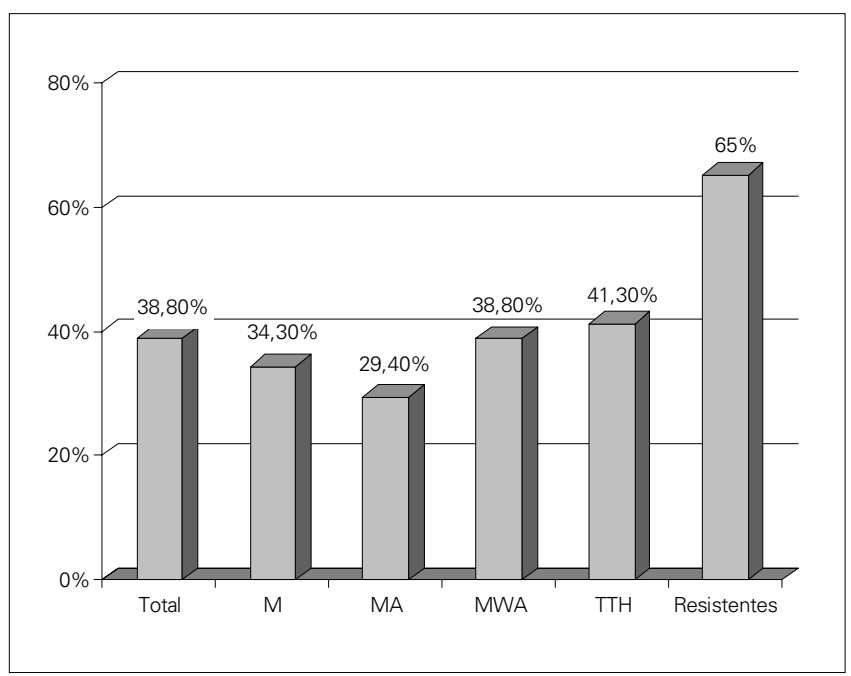

Figura 3. Prevalencia de la farmacorresistencia en el total de la muestra y en diversos subgrupos.

La prevalencia de la farmacorresistencia en la población estudiada se muestra en la figura 3. Se manifestó en 25 pacientes, 12 de los que sufrían de CTT y en 12 de los que padecían M. Por otro lado, 15 de los pacientes hipertensos también presentaron farmacorresistencia. La diferencia de prevalencia de la farmacorresistencia entre los hipertensos y los no hipertensos fue de un alto valor estadístico $(\mathrm{p}<0,001)$.

La presión de pulso media era de 49,2 $\mathrm{mmHg}$ en la muestra general, siendo de 52,8 $\mathrm{mmHg}$ entre los pacientes con farmacorresistencia; por lo tanto, la diferencia no era estadísticamente significativa.

Entre las otras enfermedades registradas de manera simultánea con la cefalea, las más frecuentes (> 3 registros) fueron: depresión y ansiedad (11 registros @ prevalencia 17,2\%), silla turca hueca, pneumoconiosis y diabetes mellitus (5 registros @ 7,8\% en cada una).

\section{DISCUSIÓN}

La literatura disponible acerca de la relación entre cefaleas e hipertensión arterial es muy escasa. Además, la mayoría de los trabajos publicados son anteriores a la actual clasificación de las cefaleas por la IHS. Asimismo, se utilizan definiciones diferentes

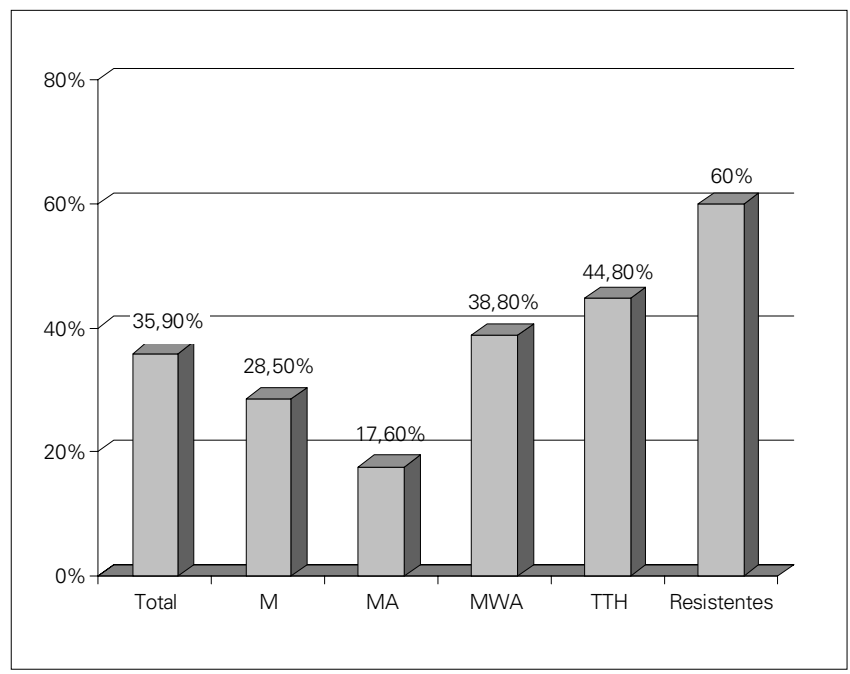

Figura 2. Prevalencia de la HTA en el total de la muestra y en diversos subgrupos.

Tabla I. Criterios de inclusión de un historial clínico en el estudio.

\begin{tabular}{l} 
Criterios de inclusión \\
\hline Edad adulta \\
\hline Tipo de cefalea identificable como migraña o CTT \\
Coincidencia documentada ${ }^{3} 1 \mathrm{~A}$ \\
Tensión arterial documentada 32 veces independientemente de la \\
cefalea aguda \\
CTT: cefalea de tipo tensional.
\end{tabular}

Tabla II. Escala JNC-VI para la clasificación de la HTA.

\begin{tabular}{ccc}
\hline Grado & TA sistólica $(\mathrm{mmHg})$ & TA diastólica $(\mathrm{mmHg})$ \\
\hline 1 (Borderline/leve) & $140-159$ & $90-99$ \\
\hline 2 (Moderada) & $160-179$ & $100-109$ \\
\hline 3 (Grave) & ${ }^{3} 180$ & ${ }^{3} 110$ \\
\hline Sistólica aislada & ${ }^{3} 140$ & $<90$ \\
\hline
\end{tabular}

para la HTA y, especialmente en los estudios más antiguos, el límite superior de la normalidad para la TA es superior al de la actual definición. Por último, en algunos trabajos la tensión arterial fue medida tan sólo una vez en cada paciente $[2,3]$.

Como los trabajos son bastante heterogéneos, tanto en la metodología como en cuanto a las poblaciones estudiadas, no sorprende que los resultados difieran tanto. La tabla III presenta de manera resumida los estudios más importantes realizados sobre esta temática. Mientras que algunos trabajos apuntan hacia una correlación entre cefalea y HTA, otros niegan cualquier asociación o mencionan solamente una asociación entre la cefalea y la HTA grave, principalmente la crisis hipertensiva. Un estudio hace referencia a una relación entre la frecuencia de cefaleas y la tensión arterial, pero considera que la naturaleza del dolor debe ser diferente en la HTA grave que en la HTA leve o moderada [4]. Según algunos autores, el valor diastólico parece estar más rela- 
Tabla III. Estudios clínicos y poblacionales sobre la asociación entre HTA y cefaleas.

\begin{tabular}{|c|c|c|}
\hline $\begin{array}{l}\text { Autor principal } \\
\text { año }\end{array}$ & Población estudiada & Resultados \\
\hline $\begin{array}{l}\text { Walker CH, } \\
1954[11]\end{array}$ & $\begin{array}{l}n^{\circ} ?, \text { población } \\
\text { general }\end{array}$ & $\begin{array}{l}\text { TA en pacientes } \\
\text { con migraña } \\
\text { con una media } \\
10 \mathrm{mmHg} \text { mayor } \\
\text { que la población } \\
\text { general }\end{array}$ \\
\hline $\begin{array}{l}\text { Moser M, } \\
1962[6]\end{array}$ & $\begin{array}{l}54 \text { pacientes } \\
\text { con ambas } \\
\text { dolencias, } 1.276 \\
\text { individuosde } \\
\text { la población general }\end{array}$ & $\begin{array}{l}35 \% \text { de los pacientes } \\
\text { con migraña presentaban } \\
\text { HTA, la cefalea mejoró } \\
\text { con el tratamiento } \\
\text { de la HTA }\end{array}$ \\
\hline $\begin{array}{l}\text { Waters WE, } \\
1971 \text { [3] }\end{array}$ & 414 & $\begin{array}{l}\text { Ninguna asociación } \\
\text { entre cefaleas y HTA }\end{array}$ \\
\hline $\begin{array}{l}\text { Badran } \mathrm{RH}, \\
1970[5]\end{array}$ & $\begin{array}{l}100 \text { con HTA, } \\
100 \text { control }\end{array}$ & $\begin{array}{l}\text { Aumento } \\
\text { de la prevalencia } \\
\text { de la cefalea sólo } \\
\text { en la HTA grave }\end{array}$ \\
\hline $\begin{array}{l}\text { Weiss NS, } \\
1972\end{array}$ & 6.672 con HTA & $\begin{array}{l}\text { Prevalencia de cefaleas } \\
25 \% \text {, sin evidencia } \\
\text { de asociación }\end{array}$ \\
\hline $\begin{array}{l}\text { Bulpitt CJ, } \\
1976 \text { [8] }\end{array}$ & $?$ & $\begin{array}{l}\text { Prevalencia } \\
\text { de las cefaleas } \\
31 \% \text { en pacientes } \\
\text { con HTA no tratada, } \\
15 \% \text { en pacientes } \\
\text { con HTA tratada } \\
\text { y en el grupo control }\end{array}$ \\
\hline $\begin{array}{l}\text { Chen TC, } \\
1987[12]\end{array}$ & $\begin{array}{l}508 \text { mujeres } \\
\text { con migraña, } \\
3.192 \text { sin migraña }\end{array}$ & $\begin{array}{l}\text { Sin evidencia } \\
\text { de asociación } \\
\text { entre HTA y migraña }\end{array}$ \\
\hline $\begin{array}{l}\text { Cooper WD, } \\
1989[4]\end{array}$ & $\begin{array}{l}11.710 \text { con HTA } \\
\text { de leve } \\
\text { a moderada }\end{array}$ & $\begin{array}{l}\text { La cefalea fue frecuente } \\
\text { y disminuyó } \\
\text { la prevalencia } \\
\text { sustancialmente con el } \\
\text { tratamiento de la HTA }\end{array}$ \\
\hline $\begin{array}{l}\text { Rasmussen, } \\
1992[2]\end{array}$ & $\begin{array}{l}974 \text { de la población } \\
\text { general }\end{array}$ & $\begin{array}{l}\text { La HTA no influyó } \\
\text { en la incidencia } \\
\text { de la cefalea }\end{array}$ \\
\hline
\end{tabular}

cionado con una mayor prevalencia de las cefaleas que el valor sistólico [2,5]. Los trabajos, en los cuales se valoró la evolución temporal, son más favorables a la existencia de una asociación entre cefalea y HTA. Básicamente, se señala una mejora en la cefalea con tratamiento de la HTA, independientemente del tratamiento hipertensivo [4,6].

Por otro lado, se verifica un aumento de la tensión arterial durante las crisis intensas de cefalea, que se considera secundario a la ansiedad [7]. De acuerdo con una antigua teoría, el paciente se queja más de cefaleas cuando es consciente de que sufre HTA, pero esta hipótesis se contradice con la evidencia de que los hipertensos tratados se quejan menos que los hipertensos no tratados $[4,6,8]$.

La conclusión de una revisión reciente fue que la asociación entre la cefalea y la HTA leve o moderada es probablemente una coincidencia, que la HTA grave o maligna está asociada a cefaleas y que la existencia de la HTA en un paciente con migraña podrá aumentar la frecuencia o intensidad de las crisis e inducir su transformación en cefalea crónica diaria (CDH) [7]. En este contexto, debemos señalar que en nuestra muestra no hubo ninguna asociación entre $\mathrm{CDH}$ y HTA.

No existen estudios acerca de la prevalencia de la HTA en la población general portuguesa. Estudios realizados en otros países indican una prevalencia del 15-50\% en la población adulta, aproximándose al 30\% cuando se aplica la escala JNC-V1 [9]. La prevalencia descrita para los pacientes con migraña es del 11-48\% [10].

Entre nuestros pacientes, la prevalencia de la HTA fue del $35,9 \%$, casi igual al resultado publicado por Moser [6]. Este valor parece ser a primera vista ligeramente elevado en relación con la población general; no obstante, debemos considerar el importante predominio femenino en la población estudiada, típico de una consulta de cefaleas. Considerando, en conjunto, el hecho de que la ratio mujeres/varones en la prevalencia de la HTA es generalmente de 0,6 , podemos concluir que la prevalencia de la HTA en nuestro colectivo es bastante elevada.

La influencia de numerosos factores en la patogenia de ambas entidades, no controlables en su totalidad en un estudio retrospectivo, impide, sin embargo, extraer conclusiones sobre una causalidad eventual de esta comorbilidad. Esta multifactorialidad se ilustra, por ejemplo, con la asociación de las cefaleas y las alteraciones metabólicas, que se produjeron en un $22 \%$ en un estudio poblacional [2]. Otro factor que puede aumentar la confusión es la potencialidad de alguna medicación antihipertensiva en producir cefaleas; parece ser que ésta se produce sobre todo en el caso de los antagonistas del calcio [4].

Ninguno de los trabajos publicados hasta la fecha aprueba la influencia de la HTA en la respuesta terapéutica de la cefalea crónica. En nuestro estudio se verificó tanto una prevalencia elevada, de manera muy significativa para la farmacorresistencia entre pacientes hipertensos, como para la HTA entre los pacientes con cefalea farmacorresistente. Noencontramos ninguna correlación entreelnivel de la HTA y la prevalencia de la farmacorresistencia; sin embargo, el reducido número total de pacientes hipertensos no permite sacar conclusiones válidas sobre los subgrupos. En uno de los trabajos revisados se sugiere también que la relación entre HTA y cefalea no sea gradual, pero que puede existir una tensión arterial umbral por encima de la cual la prevalencia de las cefaleas aumente [5].

La HTA (y también la farmacorresistencia) fue más prevalente en pacientes con CTT que en pacientes con migraña, pero esta diferencia no fue estadísticamente significativa, y se debe, con toda probabilidad, más bien a la media de edad superior en el primer grupo que a cualquier otra causa. Existe un estudio que hace referencia a la presencia de cefaleas difusas de manera más frecuente entre los hipertensos que la cefalea unilateral [6], lo cual también es cierto para la población general.

Dado el papel de la vasodilatación en la patogenia de la cefalea, principalmente en la migraña, sería razonable que existiera una correlación entre la presión de pulso y la tendencia a la cefalea. No obstante, esta relación nunca podría ser muy importante, debido a la autorregulación del tono vascular cerebral. De hecho, en nuestra muestra no se observó una diferencia significativa en la presión de pulso, ni en el contexto de la farmacorresistencia, ni en el contexto de la migraña.

Respecto a otras entidades nosológicas registradas en los pacientes estudiados, destacamos dos aspectos. Primero, la prevalencia relativamente elevada de las penumoconiosis. Ésta se debe interpretar como un hallazgo de la epidemiología regional, siendo Coimbra una región con industria cementera y de cerámica. Tan sólo cuando la enfermedad está más avanzada, se puede condicionar un estado de hipoxemia, que, a su vez, puede condicionar 
vasodilatación intracerebral y cefaleas [10]. La prevalencia de las perturbaciones psiquiátricas en esta población fue cuatro veces inferior a lo previsto. Esta diferencia se puede explicar fácilmente por el hecho de que la depresión y la ansiedad no fueron objetivos de este trabajo, y por lo tanto no fueron investigadas de manera sistemática; tan sólo se registraron cuando aparecieron explícitamente documentadas en el historial.

Aunque la multifactorialidad de la patogenia de ambas entidades nosológicas y su alta prevalencia en la población general dificulten esa afirmación, los dados obtenidos en este estudio sugieren la existencia de una asociación entre HTA y cefalea, cuando la prevalencia de la HTA en la población estudiada és más alta de los que cabría esperar en una muestra de población general con las mismas características, en lo que hace referencia a la edad y el sexo. Los mecanismos eventualmente subyacentes a esta asocia- ción está por aclarar. En los casos de aumento rápido de la tensión arterial, el mecanismo probable es una dilatación de los vasos de resistencia del cerebro, en el caso de falta de autorregulación. Sin embargo, este mecanismo no parece probable en una HTA moderada o crónica.

Podemos afirmar, no obstante, que la HTA constituye un factor agravante en la cefalea crónica y que dificulta el tratamiento eficaz de la misma. Por ello nos parece justificado incluir la medición sistemática de la tensión arterial en la consulta de pacientes con cefalea crónica y tratar la HTA cuando sea detectada.

También señalamos la necesidad de estudios prospectivos controlados, con un mayor número de pacientes, para aclarar la interacción eventualmente existente entre la HTA y la cefalea crónica, y para evaluar el beneficio que estos pacientes podrían conseguir con un control agresivo de la tensión arterial.

\section{BIBLIOGRAFÍA}

1. Silverstein S, Lipton RB, Goodsby PJ. Headache in clinical practice. Oxford: ISIS Medical Media; 1998.

2. Rasmussen BK, Olesen J. Symptomatic and nonsymptomatic headaches in a general population. Neurology 1992; 42: 1225-31.

3. Waters WE. Headache and blood pressure in the community. Br Med J 1971; 1: 142-3.

4. Cooper WD, Glover DR, Hormbrey JM, Kimber GR. Headache and blood pressure: evidence of a close relationship. J Hum Hypertens 1989; 3: 41-4.

5. Badran RH, Weir RJ, McGuinessJB. Hypertension and headache. Scand Med J 1970; 15: 48-51.

6. Moser M, Wish H, Friedman AP. Headache and hypertension. JAMA 1962; 180: 301-6.

\section{EL PAPEL DE LA HIPERTENSIÓN ARTERIAL EN LA COMORBILIDAD DE LAS CEFALEAS CRÓNICAS}

Resumen. Introducción. La literatura que hace referencia a la asociación entre cefalea e hipertensión arterial es bastante escasa, principalmente la anterior a la clasificación actual de la IHS, y se basa en métodos muy diversos, resultando por lo tanto difícil de interpretar. Objetivos. Analizar la asociación entre hipertensión arterial y los dos tipos de cefaleas crónicas y determinar cómo la hipertensión arterial puede afectar al resultado de la terapia profiláctica para el tratamiento de las cefaleas crónicas. Pacientes y métodos. Se analizaron retrospectivamente 64 historiales de pacientes externos con cefalea; los historiales fueron elegidos al azar, entre los pacientes que padecían migraña o cefalea de tipo tensional (CTT). Los pacientes se consideraron hipertensos si la presión sanguínea era superior a la normal en varias ocasiones, sin que se presentara una crisis aguda. La resistencia al tratamiento se definió como la falta de mejora en relación con el número o la intensidad de las crisis. Resultados. La edad varió entre los 18 y los 80 años, siendo la media de edad de 42,9 (DE 13,86) años. El 86\% de los pacientes eran mujeres. Veinticinco pacientes sufrían CTT y 35 migraña. La prevalencia de la hipertensión fue del 35,9\% entre todos los pacientes, $28,5 \%$ entre los pacientes con migraña y 44,8\% entre los pacientes con CTT. La prevalencia de la resistencia al tratamiento fue del 39,8, 34,3 y del $41,3 \%$, respectivamente. En cuanto a los pacientes resistentes al tratamiento, el $60 \%$ eran hipertensos y el 62,5\% de los pacientes hipertensos ofrecieron resistencia a la terapia. Conclusiones. La hipertensión arterial fue más prevalente en la muestra estudiada de lo que se cabría esperar en una muestra equivalente de la población general. La hipertensión fue significativamente más prevalente entre los pacientes resistentes a la terapia, como la resistencia al tratamiento entre los pacientes hipertensos. Por lo tanto, nuestra conclusión es que existe una comorbilidad importante entre la cefalea crónica y la hipertensión y que la hipertensión arterial complica el control de las cefaleas crónicas. [REV NEUROL 2001; 33: 119-22]

Palabras clave. Cefalea crónica. Comorbilidad. Hipertensión arterial. Resistencia a la terapia.
7. Mathew NT. Migraine and hypertension. Cephalalgia 1999; (Suppl 25): 17-9.

8. Bulpitt CJ, Dollery CT, Carna S. Change in symptoms of hypertension patients after referral to hospital clinic. Br Heart J 1976; 38: 121-8.

9. Classen M, Diehl V, Kochsiek K. Innere Medizin. 2 ed. München: Urban und Schwarzenberg; 1993.

10. Meyer JS, Teragami J, Konro S. Age-related cerebrovascular disease alters the symptomatic course of migraine. Cephalalgia 1998; 18: 202-8.

11. Walker CH. Migraine and its relationship to hypertension. Br Med J 1959; 2: 1430-3.

12. Chen TC, Leviton A, Edelstein S, Ellenberg JH. Migraine and other diseases of women of reproductive age. The influence of smoking on observed associations. Arch Neurol 1987; 44: 1024-8.

\section{PAPEL DA HIPERTENSÃO ARTERIAL}

\section{NA COMORBILIDADE DA CEFALEIA CRÓNICA}

Resumo. Introdução. A literatura relativa à associação entre a cefaleia e hipertensão arterial é escassa, na maioria é anterior à actual classificação IHS e baseada em métodos muito diversos, sendo assim difícil de interpretar. Objectivos. Analisar a associação entre hipertensão arterial e dois tipos de cefaleia crónica e determinar se e como a hipertensão arterial pode afectar o resultado da terapêutica profiláctica de cefaleias crónicas. Doentes e métodos. Foram analisados retrospectivamente 64 ficheiros dos nossos doentes ambulatórios com cefaleia, escolhidos aleatoriamente entre doentes com hemicrania ou cefaleia tipo tensão(CTT). Os doentes foram considerados como hipertensos se a pressão sanguínea fosse superior ao normal várias vezes sem uma crise aguda. A resistência ao tratamento foi definida como uma falta de benefício relativamente ao número elou à intensidade das crises. Resultados. A idade variou entre os 18 e os 80 anos, sendo a idade média 42,9 anos (DP 13,86). 86\% eram de sexo feminino. 29 doentes sofriam de CTT e 35 de hemicrania. A prevalência da hipertensão foi de 35,9\% para todos os doentes, de 28,5\% para os doentes com hemicrania, 44,8\% para os doentes com CTT. A prevalência da resistência ao tratamento foi de 39,8\%, 34,3\% e $41,3 \%$, respectivamente. $60 \%$ dos doentes resistentes à terapêutica eram hipertensos e 62,5\% dos doentes hipertensos apresentaram resistência ao tratamento. Conclusões. A hipertensão arterial foi mais prevalente na amostra em estudo do que seria esperado numa amostra equivalente da população em geral. A hipertensão foi significativamente mais prevalente entre os doentes resistentes à terapêutica, bem como a resistência ao tratamento entre os doentes hipertensos. Concluímos, portanto, que existe uma comorbilidade entre a cefaleia crónica e a hipertensão e que a hipertensão arterial complica o controlo das cefaleias crónicas. [REV NEUROL 2001; 33: 119-22]

Palavras chave. Cefaleia crónica. Comorbilidade. Hipertensão arterial. Resistência à terapêutica. 